\title{
DETERMINATION OF HEAT TRANSFER CHARACTERISTICS EMPLOYING MODIFIED MAXIMUM SLOPE METHOD FOR POROUS MEDIA HEAT EXCHANGER USING $\mathrm{Al}_{2} \mathrm{O}_{3}$-WATER NANOFLUIDS
}

\author{
Dijin $J S^{1}$, Rajkumar $M R^{2}$, and Anjan R Nair ${ }^{3, *}$ \\ ${ }^{1}$ Micro/Nanofluidics Research Lab, Department of Mechanical Engineering, College of Engineering Trivandrum, Kerala, India \\ ${ }^{2}$ Advanced Thermo Fluid Research Lab, Department of Mechanical Engineering, College of Engineering Trivandrum, Kerala, India \\ ${ }^{3}$ Micro/Nanofluidics Research Lab, Department of Mechanical Engineering, College of Engineering Trivandrum, kerala, India
}

\begin{abstract}
It has been well documented in many literature that porous media and nanofluid can augment heat transfer in many engineering system. However the combined usage of these two media has not been given much attention in literature. The objective of the present work is to experimentally investigate porous media heat exchanger using $\mathrm{Al}_{2} \mathrm{O}_{3} /$ water nanofluids. The heat transfer characteristics is determined using transient testing method wherein, only one fluid stream flows steadily through the test core, then a transient perturbation in the inlet fluid temperature is induced and the outlet fluid temperature variation is measured continuously. The measured data is evaluated using the maximum slope method to obtain the heat transfer characteristics. The results show an increase in Nusselt number values with the use of nanofluids in porous media heat exchanger compared to water.
\end{abstract}

\section{Nomenclature}

$\Delta p=$ Pressure Drop across heat exchanger $(\mathrm{Pa})$

$\mu=$ Dynamic Viscosity $(\mathrm{Kg} / \mathrm{ms})$

$\rho=$ Density $\left(\mathrm{Kg} / \mathrm{m}^{3}\right)$

$L=$ Length of heat exchanger $(\mathrm{m})$

$v_{D}=$ Duct Velocity $(\mathrm{m} / \mathrm{s})$

$k=$ Thermal conductivity $(\mathrm{W} / \mathrm{mK})$

$C_{p}=$ Specific heat at constant pressure of the fluid, $(J /$

$\mathrm{kgK}$ )

$C=$ specific heat capacity $(\mathrm{J} / \mathrm{kgK})$

$h=$ Convection heat transfer coefficient $(W / m K)$

$k=$ Thermal conductivity of the matrix material

$(W / m K)$

$M=$ Mass of the matrix $(\mathrm{kg})$

$\dot{m}=$ Mass flow rate of the fluid $(\mathrm{kg} / \mathrm{sec})$

$\mathrm{NTU}=$ Number of Transfer Units $=U A /\left(\dot{m} C_{p}\right)$

$T=$ Temperature of the fluid $(\mathrm{K})$

$T_{0}=$ Temperature of test section at time, $\xi=0(K)$

$\xi=$ Time (sec)

$\theta=$ Dimensionless temperature of the fluid at exit section, $=\left(t-t_{i n}\right) /\left(T_{o}-t_{i n}\right)$

$\zeta=$ Dimensionless time considering total mass of heat exchanger, $\zeta=\left[\dot{m} C_{p} /(M C)\right] \xi$

$\operatorname{Re}=$ Reynolds Number

$\gamma=$ Porosity

$\Phi=$ volume concentration of nanofluid

$\mathrm{w}=$ weight $(\mathrm{Kg})$

$\rho=\operatorname{density~}\left(\mathrm{Kg} / \mathrm{m}^{3}\right)$
$\mathrm{Nu}=$ Nusselt number

Subscripts

$\mathrm{f}=$ fluid

$\mathrm{s}=$ solid

eff $=$ effective

in $=$ inlet

out $=$ outlet

$\mathrm{np}=$ nanoparticle

$\mathrm{nf}=$ nanofluid

$\mathrm{bf}=$ basefluid

\section{INTRODUCTION}

In recent years, the high cost of energy and material has led to the designing of more efficient and economical heat exchangers [8-9]. A lot of research has been developed to study the different heat transfer enhancement techniques such as using fins and baffles, porous media, coiled tubes as well as adding high conductivity particles to the fluid. Porous medium in internal flows using nanofluid is believed to be one of the promising methods in this field. Porous Media Heat Exchangers [PMHE] offers a large amount of heat transfer occur within a small volume. The values of Nusselt number and convective heat transfer coefficients of systems filled with porous material is higher than those without porous material due to the high thermal conductivity of the porous matrix [11].

\footnotetext{
Corresponding author: anjanrn@gmail.com
} 
Pavel and Mohamad [1] numerically analysed the thermal performance of compact heat exchangers assuming it as a porous body. They experimentally obtained the permeability and inertia coefficients of the porous media by using the Forcheimer equation, and provided these as inputs to the numerical method. The data obtained gives the fluid temperature history at the exit of the compact heat exchanger and the time at which it occurs for the prediction of NTU using the maximum slope method proposed by Krishnakumar and Venkatarathnam [2]. They established a relationship for NTU and axial conduction parameter for various combinations of maximum slope and the time at which the maximum slope occurs. Nair and Krishnakumar [3] investigated single blow transient test on wire mesh heat exchanger using FLUENT porous media model and used maximum slope method for data evaluation. Using the data NTU values were estimated for stainless steel and galvanized iron wire meshes for two different porosities.

Nanofluid is a new kind of fluid consisting of uniformly dispersed and suspended nanometer-sized particles or fibers in fluids and has unprecedented thermal characteristics. Hwang et al. [5] experimentally investigated flow and convective heat transfer characteristics of $\mathrm{Al}_{2} \mathrm{O}_{3}$ /water nanofluid with particles varying in the range of $0.01-0.3 \%$ in a circular tube. The results shown improvement in convective heat transfer coefficient in the thermally fully developed regime. Xuan and $\mathrm{Li}$ [6] studied experimentally the convective heat transfer and flow features for $\mathrm{Cu}$-water nanofluids flowing through a straight tube under laminar and turbulent flow regimes with a constant heat flux. The experimental results showed that addition of nanoparticles into the base liquid remarkably enhanced the heat transfer performance of the base liquid.

In literature, most authors investigated the heat transfer characteristics of perforated plate heat exchanger using air and water as working fluid. In this study, transient testing was done experimentally on perforated copper plate heat exchangers using $\mathrm{Al}_{2} \mathrm{O}_{3} /$ water nanofluids. The Number of Transfer Units (NTU) in this study were obtained by the maximum slope method proposed by Krishnakumar and Venkatarathnam [4].

\section{Experiment Methodology}

Nanofluids of $0.05 \%$ volume concentrations were prepared with $\mathrm{Al}_{2} \mathrm{O}_{3}$ nanoparticles having diameter less than $50 \mathrm{~nm}$. Experiments were conducted by using water and nanofluids by varying the Reynolds numbers from 336 to 845 .

\subsection{Preparation of Nanofluid}

Nanofluid is prepared by two-step process. Nanoparticles used in this method are first produced as dry powders by chemical or physical methods. Then, the nanosized powder is dispersed into a fluid in the second processing step with the help of intensive magnetic force agitation, ultrasonic agitation or ball milling. $\mathrm{Al}_{2} \mathrm{O}_{3}$ nanoparticles were purchased from Sigma Aldrich Chemicals Bangalore. The thermophysical properties of $\mathrm{Al}_{2} \mathrm{O}_{3}$ nanoparticles are shown in Table 1 . Nanofluids of $0.05 \%$ volume concentrations were prepared by dispersing $\mathrm{Al}_{2} \mathrm{O}_{3}$ nanoparticles in de-ionized water by using probe type sonicator. Surfactants were added to avoid sedimentation of nanoparticles in the base fluid. Sodium dodecyl benzene sulphate (SDBS) surfactant with 1/4th weight of nanoparticles were added. Volume concentration of nanofluid is calculated by using the following equation:

$$
\phi=\left[\frac{\left(w_{n p} / \rho_{n p}\right)}{\left(w_{n p} / \rho_{n p}\right)+\left(w_{b f} / \rho_{b f}\right)}\right] \times 100
$$

Table 1. Thermophysical properties of $\mathrm{Al}_{2} \mathrm{O}_{3}$ nanoparticles

\begin{tabular}{|c|c|}
\hline Material & $\mathrm{Al}_{2} \mathrm{O}_{3}$ \\
\hline Diameter & $<50 \mathrm{~nm}$ \\
\hline Purity & $99 \%$ \\
\hline Density & $3970 \mathrm{Kg} / \mathrm{m}^{3}$ \\
\hline Specific heat capacity & $525 \mathrm{~J} / \mathrm{Kgk}$ \\
\hline Thermal Conductivity & $17.65 \mathrm{~W} / \mathrm{mk}$ \\
\hline
\end{tabular}

\subsection{Experiment setup}

The experiment set-up used for the investigation is shown in Fig. 1. $\mathrm{Al}_{2} \mathrm{O}_{3}$ /water nanofluid is used as the working fluid in this study. The set up consists of two circuits: cold and hot. These circuits have two centrifugal pumps to maintain flow of working fluid at the required flow rate. The fluid on the hot side is heated using a rod-type heater $(1000 \mathrm{~W})$ with an adjustable thermostat to set the required temperature.

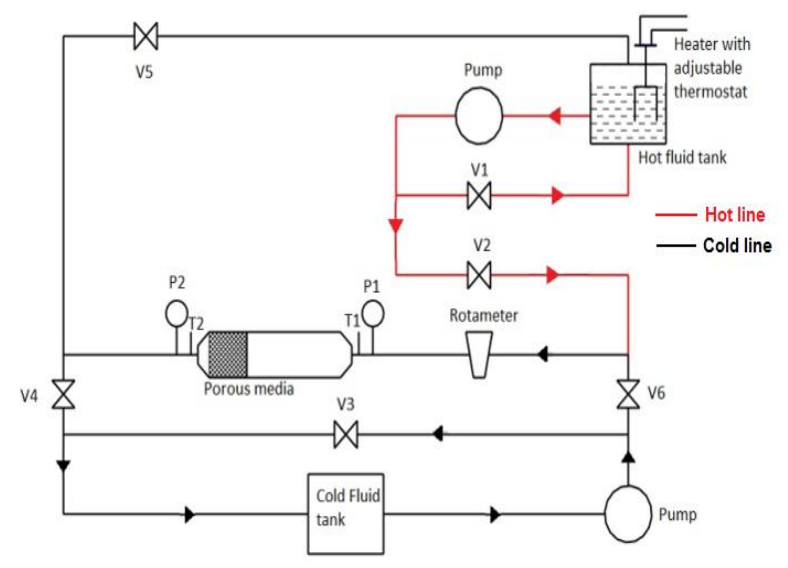

Fig. 1. Experiment Setup

The flow rate was measured using a glass type variable area flow meter (Rotameter type: 0-2 litres per minute). The temperature at the inlet and exit of the porous section was measured using calibrated $\mathrm{T}$ type thermocouples. Bourdon tube type pressure gauges with a least count $0.02 \mathrm{~kg} / \mathrm{cm}^{2}$ are placed at the inlet and exit of the porous zone to measure the pressure drop. A data acquisition system, Agilent 34972A, is used to measure the temperature variation. 


\subsubsection{Experiment procedure}

The single blow transient testing is done by changing the temperature of the nanofluid entering the porous section suddenly and observing the temperature response at the exit of the test section. Initially hot nanofluid was run through the porous section. Then a sudden drop in temperature $(\Delta \mathrm{T}=10 \mathrm{~K})$ is introduced at the inlet and the exit temperature variation for a time interval of 1 second was measured using data acquisition system. The temperature and time variables are non-dimensionlised and plotted. The maximum slope of graph and time at which it occurs is noted to obtain the values of NTU directly using the maximum slope method given in Krishnakumar and Venkatratnam [4]. The experiment was repeated for different Reynolds number and porosities $(0.2,0.32,0.46)$.

\section{Results and discussions}

The laminar flow region $(\operatorname{Re}<1000)$ was used for the study. The Fig. 2 shows the variation of dimensionless temperature and slope with dimensionless time for $\mathrm{Re}=$ 465 and porosity 0.2 . The dimensionless temperature decreases with time and reaches a steady state due to the transient perturbation given at the inlet. The value of slope increases to a maximum value and decreases. The value of maximum slope and the time at which it occurs is used to obtain NTU from literature [2].

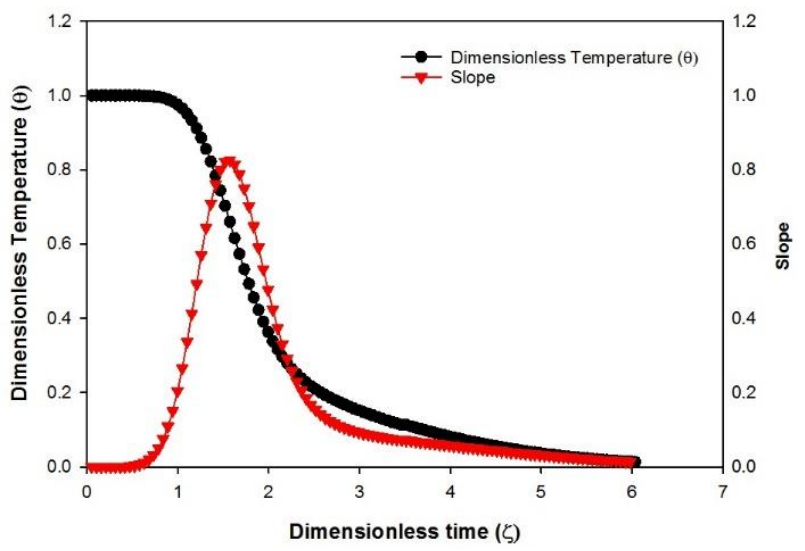

Fig. 2. Variation of dimensionless temperature and slope with time for $\mathrm{Al} 2 \mathrm{O} 3$-water nanofluid of $0.05 \%$ volume concentration at $\operatorname{Re}=465$ and porosity $\mathrm{p}=0.2$

The Fig. 3 shows the variation of NTU with Reynolds number for porosities $0.2,0.32$ and 0.46 . It is observed that the NTU values decrease with increasing Reynolds Number for both water and nanofluid. This is due to lower residence time of fluid available for heat transfer at higher flow rates. The NTU values of nanofluids were found to be higher than water for all porosities implying the increase in overall heat transfer coefficient using nanofluids.

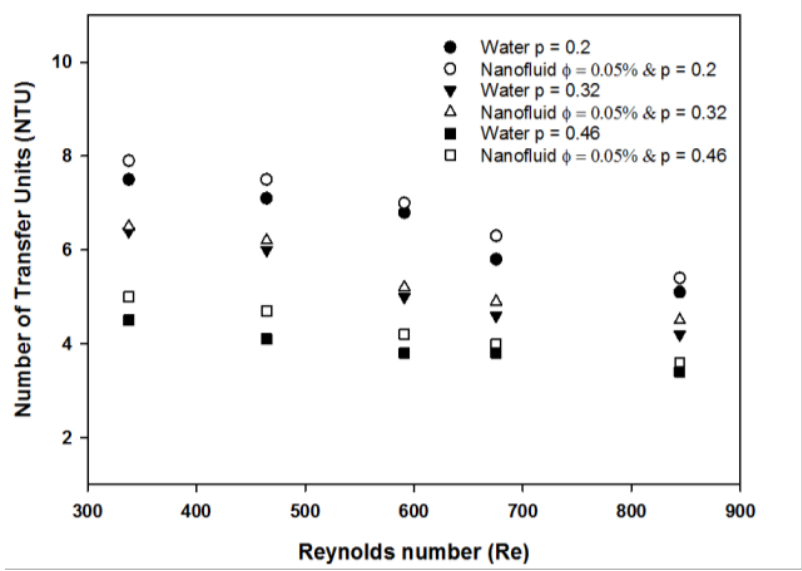

Fig. 3. Variation of NTU with Reynolds number for water and $\mathrm{Al}_{2} \mathrm{O}_{3}$-water nanofluid of $0.05 \%$ volume concentration at different porosities $(0.2,0.32,0.46)$

The Fig. 4 shows the variation of Nusselt Number with Reynolds number for different porosities. The values of Nusselt number increases with increase in Reynolds number. The Nusselt number values of nanofluids were found to be higher than water at all porosities.

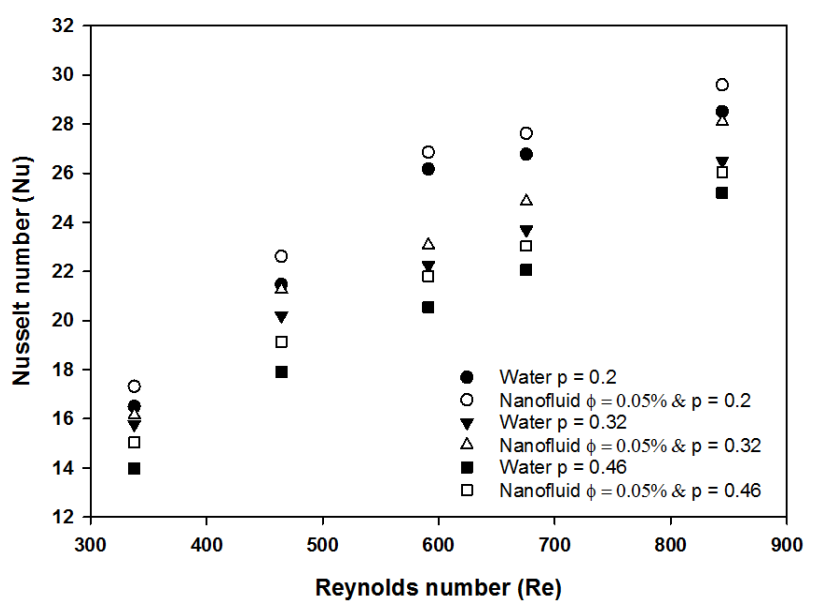

Fig. 4. Variation of Nusselt number with Reynolds number for water and $\mathrm{Al}_{2} \mathrm{O}_{3}$-water nanofluid of $0.05 \%$ volume concentration at different porosities $(0.2,0.32,0.46)$

The maximum heat transfer enhancement of $6.5 \%$ was observed for porosity 0.32 at Reynolds number 845 . It is also observed that Nusselt number values increases with decreasing porosity. This is due to increased convective heat transfer area for heat transfer available at lower porosities. 


\section{Conclusions}

Heat transfer in porous media heat exchanger using $\mathrm{Al}_{2} \mathrm{O}_{3}$ /water nanofluids of volume concentration were analysed using single blow transient testing method. The conclusions are summarized below:

(1) The NTU values decreases with increase in Reynolds number for both water and nanofluid due to lower residence time available for fluid for heat transfer.

(2) Nusselt number increases as Reynolds number increases due to higher rate of contact between fluid and porous media at higher velocities.

(3) The maximum increase in Nusselt number was $6.5 \%$ for nanofluids of volume concentration $0.05 \%$ when compared to water for porosity 0.32 at Reynolds number 845 .

(4) Nusselt number increases as porosity decreases. This is due to increased surface area for heat transfer.

\section{References}

1. Pavel, B. I., Mohamad, A. A. An experimental and numerical study on heat transfer enhancement for gas heat exchangers fitted with porous media. International Journal of Heat and Mass Transfer, 47(23), 4939-4952, (2004)

2. Krishnakumar, K., and G. Venkatarathnam. Transient testing of perforated plate matrix heat exchangers. Cryogenics 43, 101-109, (2003)

3. Nair Anjan R, Krishnakumar K. Numerical modeling of single blow transient testing of a wire screen mesh heat exchanger, 10th National conference on technological trends (2009)

4. K. Krishnakumar and G. Venkatarathnam, On the use of time at maximum slope in determining the heat transfer coefficients in complex surfaces using the single blow transient test method, International Journal of Heat Exchangers, 8, 31-38, (2007)

5. Kyo Sik Hwang, Seok Pil Jang, Stephen U.S. Choi, Flow and convective heat transfer characteristics of water-based $\mathrm{Al}_{2} \mathrm{O}_{3}$ nanofluids in fully developed laminar flow regime, International Journal of Heat and Mass Transfer 52, 193-199, (2009)

6. Xuan, Y., and Li, Q. Heat Transfer Enhancement of Nanofluids, Int. J. Heat Fluid Flow, 1(21), 58-64, (2000)

7. Sobin Alosious, Sarath S R, Anjan R Nair, K Krishnakumar, Experimental and numerical study on heat transfer enhancement of flat tube radiator using $\mathrm{Al} 2 \mathrm{O} 3$ and $\mathrm{CuO}$ nanofluids, Springer-Verlag Berlin Heidelberg (2017)

8. Mohamad, A. A. High efficiency solar air heater, Solar energy, 60(2), 71-76, (1997)

9. Bhattacharya, A., , Mahajan, R. L. Finned metal foam heat sinks for electronics cooling in forced convection, Journal of Electronic Packaging, 124(3), 155-163, (2002)
10. M.Hassan, M.Marin, AbdullahAlsharif, R.Ellahi. Convective heat transfer flow of nanofluid in a porous medium over wavy surface, Physics Letters A, 382(38), 2749-2753, (2018)

11. Fitch, John L. Method of recovering geothermal energy. U.S. Patent 3,863,709, issued February 4, (1975) 